\title{
INTRODUÇÃO E AVALIAÇÃO DE CLONES DE GOIABEIRA DE POLPA BRANCA (Psidium guajava L.) NA REGIÃO DO SUBMÉDIO SÃO FRANCISCO ${ }^{1}$
}

\author{
LUIZ GONZAGA NETO²
}

Objetivando avaliar a variabilidade genética e selecionar alternativas de cultivo para o mercado de goiaba "in natura" com polpa branca, foram introduzidas e avaliadas, preliminarmente, algumas variedades de goiaba na região do Submédio São Francisco. Foram introduzidas 22 variedades de goiabeira, provenientes do banco de germoplasma de goiabeira da empresa IPA, entre as quais algumas produtoras de fruta com polpa branca obtidas através de mudas propagadas através de enxerto, utilizando-se de quatro plantas por acesso, no espaçamento de 6,0 × 6,0 m. Nesta primeira etapa, foram avaliados os seguintes descritores: produção por planta (em kg), número de frutos colhidos e peso médio do fruto em grama. Destacaram-se as variedades Banaras e Luck Now, com potencial agronômico para serem introduzidas na área de produção comercial, com produção média (seis anos). A Banaras produziu $98,07 \mathrm{~kg} /$ planta, 813 frutos/ciclo, com peso médio de $176.97 \mathrm{~g}$, enquanto a Luck Now produziu $118.22 \mathrm{~kg}, 940$ frutos, com peso médio de 131.39 $\mathrm{g}$, respectivamente, destacando-se principalmente no descritor peso médio do fruto, que é um dos mais importantes na seleção de variedades para consumo in natura. No que se refere às características químicas, vê-se que a Seleção IPA B38.1 apresentou o maior grau Brix, $12.7^{\circ}$, enquanto, nos frutos da variedade Allahabad Safeda, foi registrado o menor, $9.8^{\circ}$.

Resumo - Objetivando avaliar a variabilidade genética e selecionar alternativas de cultivo para o mercado de goiaba "in natura" com polpa branca, foram introduzidas e avaliadas, na região do Submédio São Francisco, 22 variedades de goiaba, provenientes de um banco de gemoplasma do IPA. As mudas foram propagadas através de enxerto, utilizando-se de quatro plantas por acesso, no espaçamento de 6,0 x 6,0 m. Foram avaliados, no período de 1993 a 1998, os seguintes descritores: produção por planta (em kg), número de frutos colhidos/ciclo e peso médio do fruto em grama. Destacaram-se, no conjunto dos descritores avaliados, principalmente peso médio do fruto, um dos mais importantes descritores em goiaba para mesa, as variedades Banaras e Luck Now, com potencial agronômico para produção comercial, com produção de $98,07 \mathrm{~kg} /$ planta, 813 frutos/ciclo e peso médio do fruto de $176 \mathrm{~g} \mathrm{e} 118.22 \mathrm{~kg}, 940$ frutos, e peso médio de $131.39 \mathrm{~g}$ (média de seis anos), respectivamente. Com relação ao grau Brix, a Banaras apresentou 11,1 e a Luck Now 12,1 e uma relação SST/Acidez de 2.8 e 3.0, respectivamente.

Termos para indexação: Melhoramento, variedade, irrigação

\section{INTRODUCTION AND EVALUATION OF WHITE FLESH GUAVA (Psidium guajava L.) CLONES IN THE SUBMÉDIO SÃO FRANCISCO RIVER VALLEY}

\begin{abstract}
With the objective of evaluating the genetic variability and selecting crop alternatives for "in natura" market of white flesh guava, 22 varieties from the IPA germplasm were introduced and evaluated in the Submédio São Francisco river valley. The plants were propagated through grafting, using four plants per variety in a $6.0 \mathrm{~m} \mathrm{x} 6.0 \mathrm{~m}$ spacing. The following parameters were evaluated from 1993 to 1998: plant production ( $\mathrm{kg}$ ), number of fruits harvested per cycle and mean fruit weight (g). Among these parameters, mainly mean fruit weight (one of the most important parameters for table guava), the varieties Banaras and Luck Now showed the best performance for commercial purposes, with, respectively, $98.07 \mathrm{~kg} / \mathrm{plant}, 813$ fruits/cycle, mean fruit weight of $176 \mathrm{~g}$, and $118.22 \mathrm{~kg} /$ plant, 940 fruits/cycle and mean fruit weight of $131.39 \mathrm{~g}$. The variety Banaras had $11.1^{\circ}$ Brix and TSS/Acidity ratio of 2.8 and Luck Now had $12.1^{\circ}$ Brix and TSS/Acidity ratio of 3.0.
\end{abstract}

Index terms: Plant Breeding, variety, irrigation

\section{INTRODUÇÃO}

A agricultura irrigada do Nordeste brasileiro tem se caracterizado como uma opção de mercado do agronegócio. Dentro deste cenário, a fruticultura irrigada apresenta destacada importância na economia regional (Gonzaga Neto et al., 1999). Diversas fruteiras compõem os sistemas de produção em exploração, destacando-se, entre outras, a mangueira, a bananeira, a videira, o coqueiro e a goiabeira.

A área explorada com a cultura da goiabeira tem crescido intensivamente, estimando-se hoje uma área próxima de 4 mil hectares, somente na região do Vale do São Francisco. Apesar de a goiabeira já apresentar uma importância econômica relativa (Maia et al., 1988) e da existência de ações de pesquisas, visando a caracterizar e selecionar variedades, a maioria dessas são direcionadas para a seleção de variedades com polpa vermelha

1 (Trabalho 243/2000). Recebido: 03/10/2000. Aceito para publicação: 08/02/2002.

2 Eng $^{\circ}$. Agr ${ }^{\circ}, \mathrm{MSc}$, Embrapa - Centro de Pesquisa Agropecuária do Trópico Semi-árido (CPATSA), Caixa Postal 23, CEP 56300-000 - Petrolina - PE. Bolsista CNPq - E-mail: lgonzaga@cpatsa.embrapa.br 
(Gonzaga Neto et al., 1988). Por outro lado, as áreas comerciais em exploração com a goiabeira, no Nordeste do Brasil, são baseadas quase que exclusivamente em plantas produtoras de frutos com polpa vermelha. Isto ocorre, principalmente, devido ser aquela a coloração mais demandada pela indústria de processamento e para consumo ${ }^{2}$ in natura ${ }^{2}$. Embora a oferta de goiaba branca, no Brasil, e principalmente no mercado nordestino, seja ainda pequena, quando comparada à oferta da goiaba vermelha, observa-se a ocorrência de nichos destas no mercado, quando são disponibilizadas em casas especializadas e supermercados. Além disso, o pólo de agricultura irrigada implantado na região do Submédio São Francisco é bastante dinâmico, o que enseja uma maior demanda por outras cultivares de goiabeira.

A variedade Paluma, de polpa vermelha, é hoje a principal variedade explorada em escala comercial na região, estimando-se que ocupe mais de $90 \%$ da área plantada com goiabeira.

É importante frisar, também, que o Brasil já produziu goiaba de polpa branca para o mercado externo (Gonzaga Neto et al, 1995), podendo voltar a participar deste mercado, caso sejam selecionadas variedades adaptadas à região do Submédio São Francisco.

Existem, em exploração no Estado de São Paulo, algumas variedades de goiabeira com polpa branca (Kawati, 1997); entretanto, como existem diferenças edafoclimáticas entre aquela região e a do Submédio São Francisco é importante que haja a introdução, caracterização e avaliação desses genótipos, nas condições de cultivo do Vale, antes da implantação em escala comercial.

Paiva et al. (1997), em estudo com variedades de goiabeira, encontraram variações no período de floração e frutificação, quando cultivadas em diferentes condições climáticas, o que pode determinar o sucesso agronômico dos cultivos comerciais, consolidando ou não uma variedade em determinado ecossistema. Passos et al. (1979), em estudo realizado no Estado de Minas Gerais, destacaram que algumas variedades foram bem sucedidas, enquanto outras não foram, evidenciando assim a necessidade de avaliação das variedades em ambientes agrícolas nos quais se pretende explorar essas variedades em escala de mercado. Considerando estes aspectos, foram introduzidos, caracterizados e selecionados, sob irrigação, genótipos de goiabeira produtores de frutos com polpa branca, na região do Submédio do São Francisco.

\section{MATERIAL E MÉTODOS}

O experimento foi conduzido no campo experimental de Bebedouro, em Petrolina-PE, não tendo sido utilizado delineamento estatístico, uma vez que não era propósito do estudo comparar os clones introduzidos. A região apresenta uma altitude de $266,4 \mathrm{~m}$, com temperatura média anual de $26,3^{\circ} \mathrm{C}$ e umidade relativa do ar em torno de $68 \%$.

Os clones foram propagados através de enxertia pelo processo de borbulhia de placa em janela aberta, sendo o enxerto realizado durante o mês de outubro de 1992, em condições de campo, em porta-enxertos com oito meses de idade. Após o enxerto atingir 8 a $10 \mathrm{~cm}$ de comprimento, foram realizadas podas de formação, visando a conduzir a muda em haste única até a altura de 50 a $60 \mathrm{~cm}$ a partir do nível do solo. Nesta ocasião, procedeu-se o corte do broto terminal a fim de orientar a formação de três ou quatro ramos principais, bem distribuídos, para a formação da copa da planta. O pomar recebeu todas as práticas necessárias de irrigação, capina, adubacão e tratos fitossanitários (Gonzaga Neto \& Soares, 1995). Foram observados, preliminarmente, os seguintes parâmetros: produção por planta, número e peso médio do fruto, e coloração da polpa.

\section{RESULTADOS E DISCUSSÃO}

Considerando a análise dos dados médios de seis anos, obtidos no período de 1993 a 1998, verifica-se, na Tabela 1, que a produção média de quatro plantas variou de $98,07 \mathrm{~kg}$ na variedade Banaras até $162,22 \mathrm{~kg}$ para a seleção IPA 38.1. Gonzaga Neto et al. (1991), em estudo com algumas variedades, na região do Vale Rio Moxotó, em Ibimirim-PE, registrou para a seleção IPA 38.1, também sob condições irrigadas, em média de onze anos de produção, valor de $92 \mathrm{~kg} /$ planta. Maranca (1981) e Marteleto (1980) registraram em goiabeiras, após o sexto ano de produção, valores de 20 a $60 \mathrm{~kg} / \mathrm{planta} / \mathrm{ano}$. Destaca-se também neste estudo a produção da variedade Allabama Safed que, em média, produziu $147,14 \mathrm{~kg} /$ planta, valor que pode ser considerado bom numa planta de goiabeira conduzida com irrigação. É importante frizar que, embora todas as plantas, em todas as variedades, tenham sido propagadas vegetativamente, observaram-se variações em todos os parâmetros. Dentro da seleção IPA B38.1, a mais produtiva na média, observou-se variação de 96,12 a $180,20 \mathrm{~kg} /$ planta. Considerando que os porta-enxertos utilizados foram provenientes de propagação sexual (semente), pode-se levantar a hipótese de que a variação registrada, na performance dos clones avaliados, pode ser atribuída a possível variabilidade dos porta-enxertos. No caso da variedade Banaras, a variabilidade na produção por planta apresentou uma amplitude ainda maior, indo de 20,44 a $140,13 \mathrm{~kg} / \mathrm{planta}$, provavelmente pela mesma hipótese levantada anteriormente, uma vez que todos os genótipos em estudo receberam os mesmos tratos culturais e foram obtidos por enxertia.

Paiva et al. (1997), em estudo de competição de cultivares de goiabeira, em Eldorado do Sul, observaram, também, variações na produção em função da cultivar estudada. Teófilo Sobrinho et al. (1992), em estudo com cítrus também registraram variação com referência à performance produtiva dos clones experimentados. Isto reforça a tese da necessidade de introduzir e caracterizar genótipos de goiabeira antes de recomendá-los para uso em escala comercial.

Com referência ao número médio de frutos colhidos por planta, no período de 1993 a 1998, verifica-se, na Tabela 1, que, em média, variou de 813, na variedade Banaras, a 1594 frutos/ planta/ciclo na Seleção IPA B38.1. Gonzaga Neto et al. (1991), estudando algumas variedades na região do vale do Rio Moxotó, encontraram variações de 720 a 1922 frutos/planta/ano, registrando para Seleção IPA B38.1, na ocasião, em média de onze anos, um total de 1483 frutos/planta, valor muito próximo daquele obtido neste estudo.

Com relação, também, a esse parâmetro, observa-se que há variação dentro das mesmas variedades, o que pode ser 
TABELA 1 - Produção, em kg/Planta, número e peso médio do fruto em (g), média obtida no período 1993 a 1998, em variedades de polpa branca introduzidas e avaliadas em Petrolina-Pe.

\begin{tabular}{|c|c|c|c|c|}
\hline Varied ad es & $\mathrm{N}^{\circ}$ Planta & Prod ução kg & $\begin{array}{l}\text { Nú mero } \\
\text { Fru to }\end{array}$ & $\begin{array}{l}\text { Peso M éd io } \\
\text { Fruto }(\mathrm{g})\end{array}$ \\
\hline \multirow{4}{*}{$\begin{array}{c}\text { W h ite Selectio } \mathrm{n} \text { of } \\
\text { F ló rid a }\end{array}$} & 1 & 128,60 & 1029 & 124,97 \\
\hline & 2 & 124,62 & 1177 & 105,87 \\
\hline & 3 & 86,03 & 844 & 101,93 \\
\hline & 4 & 110,54 & 898 & 123,09 \\
\hline M éd ia & $\mathrm{X}$ & $112,44 \mathrm{c} \mathrm{v}=17.11$ & $987 \mathrm{cv}=15.05$ & $113,46 \mathrm{c} \mathrm{v}=10.31$ \\
\hline \multirow[t]{4}{*}{ S e leção IP A B 38.1} & 1 & 96,12 & 910 & 105,62 \\
\hline & 2 & 153,99 & 1799 & 85,59 \\
\hline & 3 & 180,20 & 2062 & 85,59 \\
\hline & 4 & 122,45 & 1608 & 76,15 \\
\hline M éd ia & $\mathrm{X}$ & $162,22 \mathrm{c} \mathrm{v}=26.52$ & $1594 \mathrm{cv}=30.91$ & $88,68 \mathrm{c} \mathrm{v}=14.06$ \\
\hline \multirow[t]{4}{*}{ A llahabad Safed a 2} & 1 & 146,46 & 1065 & 137,52 \\
\hline & 2 & 164,58 & 1208 & 136,24 \\
\hline & 3 & 168,44 & 1600 & 105,27 \\
\hline & 4 & 109,11 & 764 & 142,81 \\
\hline M éd ia & $\mathrm{X}$ & $147,14 \mathrm{c} \mathrm{v}=18.42$ & $1159 \mathrm{cv}=29.95$ & $130,46 \mathrm{c} \mathrm{v}=13.06$ \\
\hline \multirow[t]{4}{*}{ Banaras 3} & 1 & 130,82 & 914 & 143,12 \\
\hline & 2 & 20,44 & 162 & 126,17 \\
\hline & 3 & 140,13 & 545 & 257,11 \\
\hline & 4 & 100,92 & 556 & 181,51 \\
\hline M éd ia & $\mathrm{X}$ & $98,07 \mathrm{c} \mathrm{v}=55.13$ & $813 \mathrm{c} \mathrm{v}=56.43$ & $176,97 \mathrm{c} \mathrm{v}=32.89$ \\
\hline \multirow[t]{4}{*}{ Luck Now 49.3} & 1 & 90,06 & 796 & 113,14 \\
\hline & 2 & 109,03 & 934 & 117,60 \\
\hline & 3 & 136,92 & 719 & 190,43 \\
\hline & 4 & 136,90 & 1312 & 104,34 \\
\hline M éd ia & $\mathrm{X}$ & $118,22 \mathrm{c} \mathrm{v}=19.38$ & $940 \mathrm{c} \mathrm{v}=28.00$ & $131,39 \mathrm{c} \mathrm{v}=30.25$ \\
\hline
\end{tabular}

$\mathrm{CV}=$ coeficiente de variação

TABELA 2 - Determinação dos sólidos solúveis totais SST ( ${ }^{\circ}$ Brix), pH, acidez e relação SST/acidez em frutos de goiabeira da coleção mantida pela Embrapa Semi-Árido, Petrolina, 1999.1.

\begin{tabular}{l|c|c|c|c}
\hline \multicolumn{1}{|c|}{ V a rie d a de s } & S S T $\left({ }^{\circ}\right.$ B rix $)$ & p H & A c id e z & S S T /A c id e z \\
\hline P a tillo -2.1 & 10,8 & 4,3 & 5,0 & 2,2 \\
\hline IP A B - 38.1 & 12,7 & 4,1 & 5,8 & 2,2 \\
\hline W hite S e le c tion of F lo rid a & 11,6 & 4,3 & 4,0 & 2,9 \\
\hline L uck N ow 49.3 & 12,1 & 4,3 & 4,0 & 3,0 \\
\hline B anara s & 11,1 & 4,1 & 4,0 & 2,8 \\
\hline A lla hab ad S a fe d a & 9,8 & 4,4 & 3,0 & 3,3 \\
\hline
\end{tabular}

explicado pela mesma tese da origem do porta-enxerto, já comentada. É importante frizar que as plantas foram conduzidas sem desbaste de frutos, expondo, portanto, todo o seu potencial produtivo, o que explica, em tese, o baixo peso médio do fruto registrado em algumas plantas analisadas neste trabalho.

Considerando cada variedade, observou-se que o maior peso médio de fruto $(176,97 \mathrm{~g})$ foi registrado para a Banaras, na qual o número médio de frutos colhidos foi 813. A Banaras foi a variedade que produziu o menor numero médio de fruto. Isto possibilita levantar a hipótese de que estas variedades, se trabalhadas com desbaste, poderiam incrementar o peso médio do fruto. Gonzaga Neto et al. (1997), em trabalho conduzido com a variedade Rica, na região do Submédio São Francisco, informam que o melhor é conduzir a planta com um total de 500 frutos, por safra, de modo a obter um peso médio dentro dos padrões requeridos pelo mercado consumidor da fruta ${ }^{2}$ in natura". Observa-se, na Tabela 1, que, de modo geral, os maiores valores de peso médio do fruto foram alcançados nas variedades que produziram um menor número de frutos. Dentro desta análise, destacam-se as terceiras plantas das variedades Luck Now 49.3 e Banaras 3, as quais produziram frutos com maior peso médio: 190, 13 e 257, 11g, respectivamente. Com referência às característica químicas, verifica-se, na Tabela 2 , que o brix variou de $9,8^{0}$, na variedade Allahabad Safeda, a $12,7^{0}$, na seleção IPA B38.1. A maior relação SST/acidez, $(3,3)$ foi registrada na variedade Allahabad Safeda.

\section{CONCLUSÕES}

Considerando os resultados obtidos neste trabalho, podese concluir:

1. As variedades Luck Now 49.3 e Banaras 3 apresentam potencial agronômico que as tornam passíveis de introdução na área de produção comercial, no segmento de polpa branca. 


\section{REFERÊNCIAS BIBLIOGRÁFICAS}

GONZAGA NETO, L.;CRISTO, A.S.;CHOUDHURY, M.M. Conservação pós-colheita de frutos de goiabeira, variedade Paluma. Pesquisa Agropecuária Brasileira, Brasília, v. 34, n.1, p. 1-6,jan. 1999.

GONZAGA NETO, L.; SOARES, J.M. A cultura da goiaba. Brasília: EMBRAPA-SPI, 1995. 75p. il. (Embrapa-SPI. Coleção plantar, 27)

GONZAGA NETO, L; PEDROSA, A.C; BEZERRA, J.E.F;DANTAS, A.P; SILVA, H. M. e.Comportamento produtivo de goiabeiras no vale do rio Moxotó - Ibimirim - PE. III Seleções para consumo ao natural do fruto; onze anos de produção. In: CONGRESSO BRASILEIRO DE FRUTICULTURA, 11., 1991. Petrolina, PE. Revista Brasileira de Fruticultura, Cruz das Almas, v. 13, n.1 p. 17-24, out. 1991.

GONZAGA NETO, L.; LEODIDO, J. M;SILVA, E.E.G. da. Raleamento de frutos de goiabeira cv.Rica, em Juazeiro, BA, Brasil. Pesquisa Agropecuária Brasileira, Brasília, v.32, n.12, p. 1281-1286, dez. 1997.

GONZAGA NETO, L; ABRAMOF, L.;BEZERRA, J.E.F.; PEDROSA, A.C.; DANTAS, A. P.; SILVA, H. M. Competição de cultivares de goiabeira (Psidium guajava L.) nas regiões do vale do Rio Moxotó. II Produção: 1980-1986. Revista Brasileira de Fruticultura, Cruz das Almas, v.10, n.2, p.55-64, 1988.

KAWATI, R. Cultivares. IN: SIMPÓSIO BRASILEIRO SOBRE GOIABEIRA, 1., 1997, Jaboticabal. Anais... Jaboticabal, FCAUJ/ UNESP/FUNEP/GOIABRAS, 1997.p1-16.
MAIA, M. L; GARCIA A.E; LEITE, R.S. do S. F. Aspectos econômicos da produção e mercado. In: INSTITUTO DE TECNOLOGIA DE ALIMENTOS (Campinas-SP). Goiaba, cultura, matéria-prima, processamento e aspectos econômicos. 2. ed. rev. Ampl. Campinas, 1988, cap. 4, p. 177-224.

MARTELETO, L. Estudo da produção e dos atributos físicos e químicos de dez variedades de goiaba (Psidium guajava, $L$.) em Visconde do Rio Branco, Minas Gerais, visando ao consumo natural e à industrialização. Viçosa: U.F.V, 1980, 67p. Dissertação Mestrado.

MARANCA, G. Fruticultura comercial: mamão, goiaba, abacaxi. São Paulo. Nobel, 1981, 118p.

PAIVA, M . C. ;MANICA, I.; FIORAVANÇO, J. C.; KIST, H. Caracterização química dos frutos de quatro cultivares e duas seleções de goiabeira. Revista Brasileira de Fruticultura, Cruz das Almas, v. 19, n.1, p.57-63, abr. 1997.

SIMPÓSIO BRASILEIRO SOBRE A CULTURA DA GOIABEIRA, 1., 1997, Jaboticabal, SP. Anais...Jaboticabal: UNESP-FUNEP-FCAVJ, 1997. 184p.

PASSOS, L. P.;PINHEIRO, R. V. R. ;CASAL, I. V. W.D. I.; STRONG-HETA, P.C; CONDE, R A. Competição entre dez variedades de goiaba (Psidium guajava, L.) em Visconde do Rio Branco, MG. Revista Ceres, Viçosa, v.26, n.147, p.417-433, 1979

TEÓFILO SOBRINHO, J.; POMPEU JÚNIOR, J.; FIGUEIREDO, J. O. de; DEMÉTRIO, C. G. B.; BARBIN, D. Competição entre quinze clones de laranjeira - pêra, ,na região de Limeira. Revista Brasileira de Fruticultura, Cruz das Almas, v. 14, n. 1, p. 41-48, 1992. 